\title{
CRYSTAL STRUCTURE OF ICE ACCRETED ON AN ICE SUBSTRATE
}

\section{Abstract}

by

O. NASELlo

FAMAF - Universidad Nacional de Cordoba, Argentina

L. LEVI

Consejo Nacional de Investiagaciones Cientificias y Técnicas (CONICET)

\section{F. PRODI}

FISBAT - C.N.R. - Reparto Nubi e Precipitazioni, Bologna, Italy

The analysis of ice deposits grown by accretion is usually carried out by examining zones of the normal sections of the accreted cylinder, where the surface temperature $T_{8}$ has reached its stationary value. Thus, the first 1-2 $\mathrm{mm}$ accretion layers are not examined. However, the characteristics of these layers could yield information on mechanisms operating during the accretion process. Consequently, in the present work, the initial stage of the accretion process has been studied by analysing the rime structure formed on a prismatic substrate, exposed during a few seconds to a droplet flux, and the structure of the first $0.5-1 \mathrm{~mm}$ layers of large accretions, $1-2 \mathrm{~cm}$ thick.

The experiments were carried out at different air temperatures $\mathrm{T}_{\mathrm{a}}$, in an icing wind tunnel, at $30 \mathrm{~m} / \mathrm{s}$. When the prism substrate was used, $T$ was regulated by an electrical resistance, keeping $\mathrm{T}_{\mathrm{a}}^{\mathrm{a}}>\mathrm{T}_{\mathrm{a}}$. When cylindrical accretions were grown, $T_{a}$ increased from $T_{s}=T_{a}$ to a stationary value, determined by heat balance.

(1) When the prism substrate was used, the results showed that, for droplet injection times $t \geqslant 10 \mathrm{~s}$, the exposed surface was completely covered by new crystals. When $t_{a}=-18,-20^{\circ} \mathrm{C}, \mathrm{T}_{\mathrm{s}}=-2,-4^{\circ} \mathrm{C}$, the crystal size increased with injection time, the mean area, at $t=60 \mathrm{~s}$ being $\delta=0.01 \mathrm{~mm}^{2}$. The accretion thickness was in this case about $1 \mathrm{~mm}$. For these crystals, the frequency distributions for the angle $y$ between the $c$-axes and the surface normal were rather scattered at $t \leqslant 15 \mathrm{~s}$, but presented two maxima, one corresponding to the prismatic orientation of the substrate, the other to a new orientation with the c-axis normal to the surface. For larger $t$ the latter orientation prevailed.

(2) Analysis of the first accretion layers showed that the crystal size depended on $T_{a}, T_{8}$ but was on average comparable to that found in (1) for injection times of $60 \mathrm{~s}$. The crystals were radially elongated and no evidence was observed of the smaller crystals, which should have been formed at the very beginning of the accretion. The frequency distributions of the angle $\eta$ between the radial direction and the projections of the c-axes on the section plane showed maxima that, for $\mathrm{T}_{\mathrm{a}}$ varying between -18 and $-24^{\circ} \mathrm{C}$, were mostly located near $30-40^{\circ}$. In the stationary zone, the same accretions presented frequency distributions with maxima at smaller angles.

Comparison of the results in (1) and (2) suggests that, when supercooled droplets freeze superposed to each other on a substrate, the preferred orientation is rapidly but not immediately established. A mechanism of nucleation and subsequent orientation selection must consequently operate. It must be noted, however, that the randomly oriented small crystals, possibly formed at the very beginning of the process, usually do not appear when the first layers of large accretions are analysed. This may be due to grain growth occurring in the first layers, during the formation of the subsequent accretion. On the other hand, the differences observed between the location of the frequency distributions maxima for the $y$ or $\eta$ angles, in the initial and stationary accretion structure, may be due to the lower initial value of $\mathrm{T}_{\mathrm{s}}$.

This result confirms that a good correlation exists between the c-axis orientation and the $T, T$ pair, which is independent of surface structure. Actually, as surface roughnesses progressively develop during growth, they could only be responsible for the observed variations of the c-axis orientation, if the maxima of the $y$ or $\eta$ distributions corresponded to angles increasing with the radial distance from the accretion centre.

Concluding that the relation between the position of the maxima in the $y$ or $\eta$ distributions, and values of $T_{a}$, $T_{b}$, is intrinsically related to the growth mode of ice, the mechanisms of orientation selection operating during droplet freezing will be discussed, taking into account the ice crystals' growth habits as a function of the temperature. 